\title{
The sector decomposition approach to real radiation at NNLO
}

\author{
G. Heinrich ${ }^{\mathrm{a}}$ *

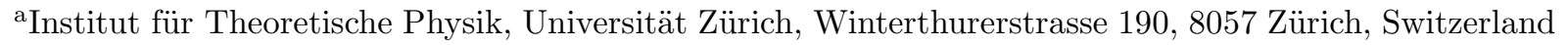

A method based on sector decomposition is briefly described which is suitable for the calculation of the double real radiation part of $e^{+} e^{-} \rightarrow 3$ jets at next-to-next-to-leading order in a fully differential way.

\section{Introduction}

The successful comparison with theory of a wealth of data collected at high energy collider experiments in the past decades largely relied on the calculation of radiative corrections. Calculating at next-to-leading order (NLO) accuracy in perturbation theory is in many cases sufficient to match the experimental precision. However, there are also prominent exceptions where the NLO predictions still suffer from large uncertainties, or where the experimental precision is extremely high. The latter is the case for measurements of jet rates and shape observables in $e^{+} e^{-}$annihilation, which for example allow for a precise determination of the strong coupling constant $\alpha_{s}$. As only NLO predictions are available for $e^{+} e^{-} \rightarrow 3$ jets, the corresponding LEP measurements have not been included in the current world average for $\alpha_{s}$. A future International Linear Collider (ILC) will allow for precision measurements at the per-mille level, which offer the possibility of a determination of $\alpha_{s}$ with unprecedented precision. However, this will only be possible if theoretical predictions for jet rates and related observables at NNLO accuracy are available.

The calculation of $e^{+} e^{-} \rightarrow 3$ jets at order $\alpha_{s}^{3}$ requires the calculation of virtual two-loop corrections combined with a $1 \rightarrow 3$ parton phase space, one-loop corrections combined with a $1 \rightarrow 4$ parton phase space where one parton can become soft and/or collinear ("theoretically unresolved"),

\footnotetext{
*To appear in the proceedings of the 7th International Symposium on Radiative Corrections (RADCOR05), Shonan Village, Japan, 2005.
}

and the tree level matrix element squared for $1 \rightarrow 5$ partons where up to two partons can become unresolved. After the virtual two-loop corrections have become available 1, the stumbling block now is given by the double real radiation part, where the unresolved particles lead to a complicated infrared singularity structure which manifests itself upon phase space integration. These singularities have to be subtracted and cancelled with the ones from the virtual contributions before a Monte Carlo program can be constructed. To achieve this task, two different approaches have been followed, one relying on the manual construction of an analytical subtraction scheme 23456789], the other one relying on sector decomposition 101112131415161718. The application of sector decomposition 1920 , 21. to NNLO phase space integrals has first been proposed in [10]. Subsequently, it has been applied to calculate inclusive phase space integrals for $e^{+} e^{-} \rightarrow 2$ jets at NNLO 111314 . The combination of the sector decomposition approach with a measurement function first has been presented in 12, and already lead to a number of important results 151617. Its application to the double real radiation part of $e^{+} e^{-} \rightarrow 3$ jets at order $\alpha_{s}^{3}$ [18] represents a new degree of complexity and therefore will be a crucial test of the potential of this method.

The main features of the two approaches are the following: Within the methods based on the explicit construction of a subtraction scheme, the subtraction terms are integrated analytically over the unresolved phase space such that the pole coefficients are obtained in analytic form. This requires appropriate phase space factorisation and 
subtraction terms which are simple enough to be integrated analytically in $D=4-2 \epsilon$ dimensions. The method naturally leads to a close to minimal number of subtraction terms, and observing the way how the poles of the different contributions cancel allows insights into the infrared structure of QCD.

In the sector decomposition approach, the poles are isolated by an automated routine and the pole coefficients are integrated numerically. The advantages of this approach reside in the fact that the extraction of the infrared poles is algorithmic, and that the subtraction terms can be arbitrarily complicated as they are integrated only numerically. On the other hand, the algorithm which isolates the poles increases the number of original functions and in general does not lead to the minimal number of subtraction terms, thus producing rather large expressions.

\section{The Method}

The universal applicability of sector decomposition goes back to the fact that it acts in parameter space by a simple mechanism. The parameters can be Feynman parameters in the case of multi-loop integrals, or phase space integration variables, or a combination of both. In the following, the working mechanism of sector decomposition will be outlined only briefly, details can be found in 2118 .

An overlapping singularity in parameter space is of the type

$$
I=\int_{0}^{1} d x \int_{0}^{1} d y x^{-1-\epsilon}(x+y)^{-1},
$$

where a naive subtraction of the singularity for $x \rightarrow 0$ fails. To solve this problem, one can split the integration region into sectors where the variables $x$ and $y$ are ordered by multiplying (11) with unity in the form $[\underbrace{\Theta(x-y)}_{(a)}+\underbrace{\Theta(y-x)}_{(b)}]$. Then the integration domain is remapped to the unit cube: after the substitutions $y=x t$ in sector (a) and $x=y t$ in sector $(\mathrm{b})$, one has

$$
I=\int_{0}^{1} d x x^{-1-\epsilon} \int_{0}^{1} d t(1+t)^{-1}
$$

$$
+\int_{0}^{1} d y y^{-1-\epsilon} \int_{0}^{1} d t t^{-1-\epsilon}(1+t)^{-1}
$$

where the singularities are now factorised. For more complicated functions, several iterations of this procedure may be necessary, but it is easily implemented into an automated subroutine. Once all singularities are factored out, they can be subtracted and the result can subsequently be expanded in $\epsilon$. Note that the subtractions of the pole terms naturally lead to plus distributions 12 by the identity

$$
\begin{gathered}
x^{-1+\kappa \epsilon}=\frac{1}{\kappa \epsilon} \delta(x)+\sum_{n=0}^{\infty} \frac{(\kappa \epsilon)^{n}}{n !}\left[\frac{\ln ^{n}(x)}{x}\right]_{+}, \text {where } \\
\int_{0}^{1} d x f(x)[g(x) / x]_{+}=\int_{0}^{1} d x \frac{f(x)-f(0)}{x} g(x) .
\end{gathered}
$$

In this way, a Laurent series in $\epsilon$ is obtained, where the pole coefficients are sums of finite parameter integrals which can be evaluated numerically.

For the numerical evaluation of loop integrals it has to be assured that no integrable singularities are crossed which spoil the numerical convergence. For integrals depending only on a single scale, which can be factored out, this does not pose a problem at all. For integrals with more than one scale, like for example two-loop box diagrams, the situation is more difficult, but in the case of $e^{+} e^{-}$annihilation to massless final state particles, evaluation over the whole physical region is possible, as the kinematics of these processes is such that the Mandelstam variables are always non-negative.

\section{Application to $e^{+} e^{-} \rightarrow 3$ jets at NNLO}

In order to focus on a concrete example of phenomenological relevance, we will discuss the application of sector decomposition to the calculation of $e^{+} e^{-} \rightarrow 3$ jets at NNLO in the following. Although the virtual contributions are not the main subject of this talk, they will be commented on briefly. 


\subsection{Double virtual and mixed real-virtual contributions}

The contributions to the amplitude which involve virtual integrals are composed of the twoloop (and one-loop times one-loop) corrections combined with a $1 \rightarrow 3$ particle phase space, and the mixed real-virtual contributions where oneloop corrections are combined with a $1 \rightarrow 4$ particle phase space with up to one unresolved particle. In both cases, sector decomposition for loop integrals 21] can serve to extract the poles in $1 / \epsilon$.

In the case of the two-loop integrals, the result will only depend on the invariants $y_{1}=$ $s_{12} / q^{2}, y_{2}=s_{13} / q^{2}$ and $y_{3}=s_{23} / q^{2}$, where $q^{2}$ is the invariant mass of the $e^{+} e^{-}$system and $\sum_{i=1}^{3} y_{i}=1$. However, as the full two-loop matrix element is known analytically [1], this part could also be taken from the literature, thus gaining a considerable amount of CPU time. The subsequent phase space integration over the $y_{i}$ is trivial, and the 3 -jet measurement function will make sure that all events where a singular limit $y_{i} \rightarrow 0$ is approached will be rejected.

In the case of the one-loop contributions, the most complicated objects will be 5-point integrals with one off-shell external leg. Sector decomposition will lead to a result in terms of five independent scaled Mandelstam invariants $y_{i}$. This result has to be calculated up to order $\epsilon^{2}$, as it will be combined with the $1 \rightarrow 4$ parton phase space where one parton can become unresolved, leading to $1 / \epsilon^{2}$ poles. This does in principle not constitute a problem, as the expansions to higher order in $\epsilon$, as well as $1 \rightarrow 4$ parton phase space integrals, are well under control within sector decomposition. It is also possible to do parts of the loop integrations analytically to achieve a form which is suitable for subsequent sector decomposition 14 17. However, these contributions have not yet been implemented into the Monte Carlo program.

\subsection{Real radiation at NNLO}

In [18, the method based on sector decomposition is for the first time applied to extract the poles appearing in massless $1 \rightarrow 5$ particle integrals. The correctness of the results for the integrals over the $1 \rightarrow 5$ particle phase space can be checked by exploiting the fact that the sum over all cuts of a given (UV renormalised) diagram must be infrared finite. This is shown in Figure 1 for a sample diagram: Summing over all cuts of this diagram and performing UV renormalisation, we obtain the condition

$T_{1 \rightarrow 5}+z_{1} T_{1 \rightarrow 4}+z_{2} T_{1 \rightarrow 3}+z_{3} T_{1 \rightarrow 2}=$ finite,

where $T_{1 \rightarrow i}$ denotes the diagram with $i$ cut lines.

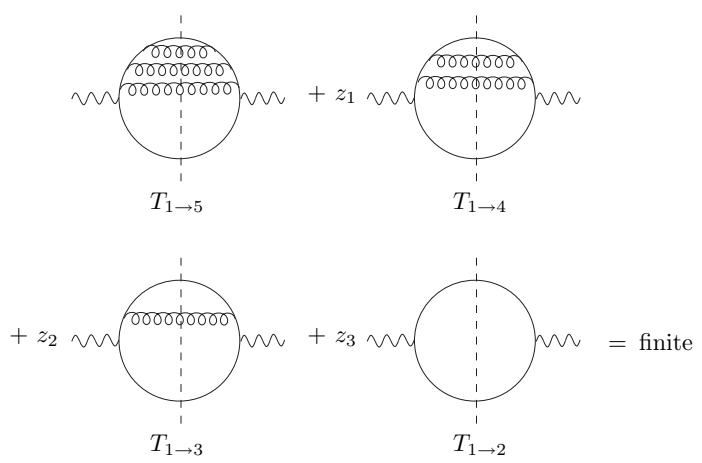

Figure 1. Cancellation of IR divergences in the sum over all cuts of the renormalised graph

The renormalisation constants $z_{i}$ (in Feynman gauge) are given by 1318

$$
\begin{aligned}
& z_{1}=C_{F} \frac{\alpha_{s}}{4 \pi} \frac{1}{\epsilon}, z_{2}=C_{F}^{2}\left(\frac{\alpha_{s}}{4 \pi}\right)^{2}\left(\frac{1}{2 \epsilon^{2}}-\frac{1}{4 \epsilon}\right) \\
& z_{3}=C_{F}^{3}\left(\frac{\alpha_{s}}{4 \pi}\right)^{3}\left(\frac{1}{6 \epsilon^{3}}-\frac{1}{4 \epsilon^{2}}+\frac{1}{6 \epsilon}\right) .
\end{aligned}
$$

The important new ingredient in eq. (3) is the calculation of $T_{1 \rightarrow 5}$. The sector decomposition method leads to 18

$$
\begin{aligned}
T_{1 \rightarrow 5}= & -C_{F}^{3}\left(\frac{\alpha_{s}}{4 \pi}\right)^{3} T_{1 \rightarrow 2}\left\{\frac{0.16662}{\epsilon^{3}}\right. \\
& +\frac{1}{\epsilon^{2}}\left[1.4993-0.4999 \log \left(\frac{q^{2}}{\mu^{2}}\right)\right] \\
& +\frac{1}{\epsilon}\left[5.5959-4.4978 \log \left(\frac{q^{2}}{\mu^{2}}\right)\right. \\
& \left.\left.+0.7498 \log ^{2}\left(\frac{q^{2}}{\mu^{2}}\right)\right]+ \text { finite }\right\},
\end{aligned}
$$

where the numerical accuracy is $1 \%$. The expressions entering eq. (3) for $i<5$ combine to [18]

$$
z_{1} T_{1 \rightarrow 4}+z_{2} T_{1 \rightarrow 3}+z_{3} T_{1 \rightarrow 2}=
$$




$$
\begin{aligned}
& C_{F}^{3}\left(\frac{\alpha_{s}}{4 \pi}\right)^{3} T_{1 \rightarrow 2}\left\{\frac{1}{6 \epsilon^{3}}+\frac{1}{2 \epsilon^{2}}\left[3-\log \left(\frac{q^{2}}{\mu^{2}}\right)\right]\right. \\
& +\frac{1}{\epsilon}\left[5.61-\frac{9}{2} \log \left(\frac{q^{2}}{\mu^{2}}\right)+\frac{3}{4} \log ^{2}\left(\frac{q^{2}}{\mu^{2}}\right)\right] \\
& + \text { finite }\}
\end{aligned}
$$

We can see that the poles in (6) are exactly cancelled by the 5-parton contribution (5) within the numerical precision.

\subsection{Differential Monte Carlo program}

Although the sector decomposition approach is considered to be a "numerical method", as the pole coefficients are only calculated numerically, the isolation of the poles is an algebraic procedure, leading to a set of finite functions for each pole coefficient as well as for the finite part. This feature allows the inclusion of any (infrared safe) measurement function, at the level of the final Monte Carlo program, which means that the subtractions and expansions in $\epsilon$ do not have to be redone each time a different observable is considered. However, in most cases of physical relevance, the measurement function is such that it would prevent certain poles from arising at all if it were included in the $\epsilon$-expansion. For example, poles associated with a 2-jet configuration, where 3 of the 5 final state particles become theoretically unresolved, would be avoided ab initio by a 3 -jet measurement function. As extra poles are rather costly in what concerns the creation of a large number of terms, it would therefore be desirable not to produce the terms associated with the subtraction of these poles at all in the $\epsilon$-expansion. In short, there is a certain dilemma between maximizing the flexibility to include any measurement function only at the stage of the final Monte Carlo program and limiting the size of the produced expressions. For $e^{+} e^{-} \rightarrow 3$ jets at NNLO, limiting this size is one of the most important issues. Therefore, this dilemma has been solved by including some "preselection rules" in the $\epsilon$-expansion which reject configurations which will surely be 2 -jet configurations. In the example shown here, this can be achieved by introducing a cut parameter $y^{\text {th }}$ - which must be smaller than any possible experimental resolution parameter $y^{\text {cut }}-$ for the variable $s_{1345}$, as $s_{1345} \rightarrow 0$ always corresponds to a 2-jet configuration. Note that this procedure does not introduce any dependence on $y^{\text {th }}$ in the matrix element, its only effect is to prevent the $\epsilon$-expansion subroutine from doing subtractions for $s_{1345} \rightarrow 0$. In this way, one can reduce the size of the expressions considerably without loosing any flexibility in what concerns the actual measurement function to be included later. In fact, the architecture of the program described in 18 is the one of a partonic event generator, such that fully differential information about the final state is available. This architecture is independent of the actual matrix element, so the toy matrix element used in [18] and in this article can finally be replaced by a different module for the full matrix element, without destroying the property of providing the four-momenta of all final state particles.

As an example, 3-, 4- and 5-jet rates using the JADE algorithm [22] are shown in Fig. 2] based on a toy matrix element built from the graphs shown in Fig. [1]

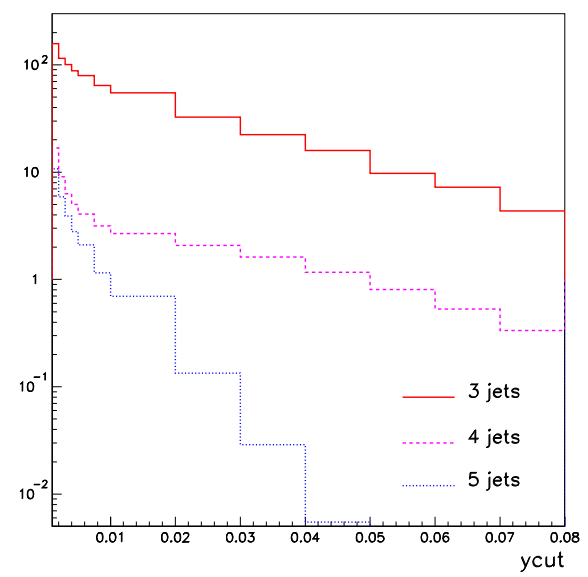

Figure 2. $3-, 4^{-}$and 5-jet rates at order $\alpha_{s}^{3}$ for the toy matrix element

\section{Outlook}

The sector decomposition approach to the calculation of NNLO cross sections is a very powerful tool, especially in what concerns the double real radiation part, as it requires neither the manual 
construction of subtraction terms, nor the factorisation of the phase space and the analytic integration of the subtraction terms in the singular limits. In [18, the method is applied for the first time to a massless $1 \rightarrow 5$ process. The code is constructed as a partonic event generator, which means that fully differential information about the final state particles is at one's disposal.

A disadvantage of the sector decomposition approach is given by the fact that it produces very large expressions, as in each decomposition step, the number of original functions increases. Therefore, CPU time is an issue for the treatment of processes with a large number of massless particles in the final state, as for example $e^{+} e^{-} \rightarrow$ 3 jets at NNLO. However, the method sketched here relies on a division of the amplitude squared into different "topologies" corresponding to different classes of denominator structures, such that the problem is naturally split into smaller subparts. Further, the size of the expressions can be reduced by including information about physical limits already at the level of the $\epsilon$-expansion, without loosing any flexibility in what concerns the definition of observables at the Monte Carlo level.

As the method is based on a universal algorithm acting on integration variables, it will surely see a number of interesting applications in the future.

\section{Acknowledgements}

I would like to thank the organizers of RADCOR05 for the interesting conference and nice atmosphere. This work was supported in part by the Swiss National Science Foundation (SNF) under contract number 200020-109162.

\section{REFERENCES}

1. L. W. Garland, T. Gehrmann, E. W. N. Glover, A. Koukoutsakis, and E. Remiddi, Nucl. Phys. B627, $107 \quad$ (2002), hep-ph/0112081 and Nucl. Phys. B642, 227 (2002), hep-ph/0206067

2. D. A. Kosower, Phys. Rev. D67, 116003 (2003), hep-ph/0212097

3. S. Weinzierl, JHEP 03, 062 (2003), hep-ph/0302180

4. A. Gehrmann-De Ridder, T. Gehrmann, and E. W. N. Glover, Nucl. Phys. B691, 195 (2004), hep-ph/0403057

5. W. B. Kilgore, Phys. Rev. D70, 031501 (2004), hep-ph/0403128

6. S. Frixione and M. Grazzini, JHEP 06, 010 (2005), hep-ph/0411399

7. A. Gehrmann-De Ridder, T. Gehrmann, and E. W. N. Glover, Nucl. Phys. Proc. Suppl. 135, 97 (2004), hep-ph/0407023

8. G. Somogyi, Z. Trocsanyi, and V. Del Duca, JHEP 06, 024 (2005), hep-ph/0502226

9. A. Gehrmann-De Ridder, T. Gehrmann, and E. W. N. Glover, JHEP 09, 056 (2005), hep-ph/0505111.

10. G. Heinrich, Nucl. Phys. Proc. Suppl. 116, 368 (2003), hep-ph/0211144

11. A. Gehrmann-De Ridder, T. Gehrmann, and G. Heinrich, Nucl. Phys. B682, 265 (2004), hep-ph/0311276.

12. C. Anastasiou, K. Melnikov, and F. Petriello, Phys. Rev. D69, 076010 (2004), hep-ph/0311311

13. T. Binoth and G. Heinrich, Nucl. Phys. B693, 134 (2004), hep-ph/0402265

14. G. Heinrich, Nucl. Phys. Proc. Suppl. 135, 290 (2004), hep-ph/0406332

15. C. Anastasiou, K. Melnikov, and F. Petriello, Phys. Rev. Lett. 93, 032002 (2004), hep-ph/0402280

16. C. Anastasiou, K. Melnikov, and F. Petriello, Nucl. Phys. B724, 197 (2005), hep-ph/0501130

17. C. Anastasiou, K. Melnikov, and F. Petriello, (2005), hep-ph/0505069

18. G. Heinrich, hep-ph/0601062.

19. K. Hepp, Commun. Math. Phys. 2, 301 (1966).

20. M. Roth and A. Denner, Nucl. Phys. B479, 495 (1996), hep-ph/9605420

21. T. Binoth and G. Heinrich, Nucl. Phys. B585, 741 (2000), hep-ph/0004013

22. JADE collaboration, S. Bethke et al., Phys. Lett. B213, 235 (1988). 